\title{
THE INCORPORATION OF PLASMA FREE FATTY ACIDS INTO PLASMA TRIGLYCERIDES IN MAN *
}

\author{
By S. J. FRIEDBERG, R. F. KLEIN, D. L. TROUT, M. D. BOGDONOFF AND \\ E. H. ESTES, JR.
}

\author{
(From the Central Reference Laboratory and Radioisotopes Unit, Veterans Administration Hos- \\ pital, and Department of Medicine, Duke University Medical Center, \\ Durham, N.C.)
}

(Submitted for publication January 26, 1961 ; accepted June 8, 1961)

Plasma triglycerides (neutral fat) may be divided into 1) that portion which is present during the fasting state and 2) that which, after ingestion of fat, is added to and temporarily augments the fasting level of triglycerides.

The present investigation deals with the relationship of plasma free fatty acids (plasma FFA) to fasting plasma triglycerides (plasma TG) in man. From these observations a quantitative assessment of the amount of plasma FFA converted to plasma TG will be presented. The data also permit calculations which suggest that plasma FFA may be the major source of fasting plasma TG.

\section{METHODS}

Two types of studies were carried out in male patients without known abnormalities of fat metabolism.

1. Non-reinjection experiments. After an overnight fast of 15 hours, 9 subjects were given 0.004 to $0.008 \mathrm{mc}$ albumin-bound palmitic acid-1- $\mathrm{C}^{\mathbf{1 4}}$ intravenously either by single injection or by 2 -hour constant infusion. Two individuals received $0.00585 \mathrm{mc}$ of albumin-bound oleic acid-1-C $\mathrm{C}^{14}$ and another $0.00597 \mathrm{mc}$ of stearic acid-1- $\mathrm{C}^{14}$. During the day of the study the subjects were permitted no breakfast, a nonfat lunch, and an unrestricted supper. Venous blood samples were drawn at appropriate intervals over a period of 24 hours after administration of fatty acid $\mathrm{C}^{\mathbf{1}}$ for determination of neutral fat activity. Two-ml aliquots of plasma were extracted by the method of Dole (1). Eight $\mathrm{ml}$ of the upper phase, representing $1.85 \mathrm{ml}$ of plasma, was transferred to a separate tube. Ten $\mathrm{ml}$ of $0.1 \mathrm{~N} \mathrm{NaOH}$ in 50 per cent ethanol was added, according to the method of Borgström (2), in order to

* This investigation was supported in part by a grant from the Life Insurance Medical Research Fund; by a research grant $\mathrm{H}-4807$; a training grant, HTS-5369, from the National Heart Institute, Public Health Service; a research grant, A-4535-MET, from the National Institutes of Health, Public Health Service; a research grant for July 1960-61, from the North Carolina Heart Association; and by the Regional Center for the Study of Aging, Duke University. remove fatty acids from the upper phase. The upper phase and its contents were then transferred quantitatively to liquid scintillator vials, dried with a stream of warm air from a hair dryer, and redissolved in $5 \mathrm{ml}$ of phosphor solution for counting in a Packard Tri-Carb liquid scintillation spectrometer. Along with plasma glycerides, the extracts contained cholesterol, cholesterol esters and a small amount of phospholipid. These would also be expected to become labeled; therefore, it was necessary to determine the amount of activity in the various plasma lipids present in the extracts at various times after injection of $\mathrm{C}^{14}$-labeled fatty acids. This was done by means of silicic acid chromatography using the method of Hirsch and Ahrens (3) without modification. Plasma palmitic acid-1- $\mathrm{C}^{\mathbf{1 4}}$ activity was measured as previously described (4).

The experimental plan required a method which would permit easy and reproducible determinations on a large number of samples. The method selected for measuring plasma TG activity described in the preceding paragraph is based on the facts that: 1) the label in the extracts appeared almost exclusively in TG in the early hours of the procedure, and 2) negligible activity was present in cholesterol, cholesterol esters and phospholipid until at least 4 hours had elapsed. Furthermore, although significant activity appeared in phospholipids, only a small fraction of this phospholipid activity was present in the upper phase extracts, and activity in free cholesterol was minimal. The data justifying the method appear below.

Total plasma triglycerides were determined by the method of Van Handel and Zilversmit (5), plasma FFA by the method of Dole (1) and esters of carboxylic acids by the method of Rapport and Alonzo (6). Free cholesterol was precipitated as the digitonide and redissolved in methanol for liquid scintillation counting. Samples of expired air were analyzed for $\mathrm{C}^{14} \mathrm{O}_{2}$ activity by trapping in Hyamine hydroxide according to the method of Fredrickson and Ono (7).

2. Reinjection experiments. In order to establish with greater certainty the validity of using the rate of disappearance of TG label observed during non-reinjection experiments to measure fasting plasma TG turnover rate, a patient, terminally ill with degenerative cerebellar disease, was given $30 \mu \mathrm{c}$ palmitic acid-1- $\mathrm{C}^{\mathbf{1 4}}$ intravenously. Blood samples were drawn at intervals for determination of triglyceride activity. One hour after injection of the 

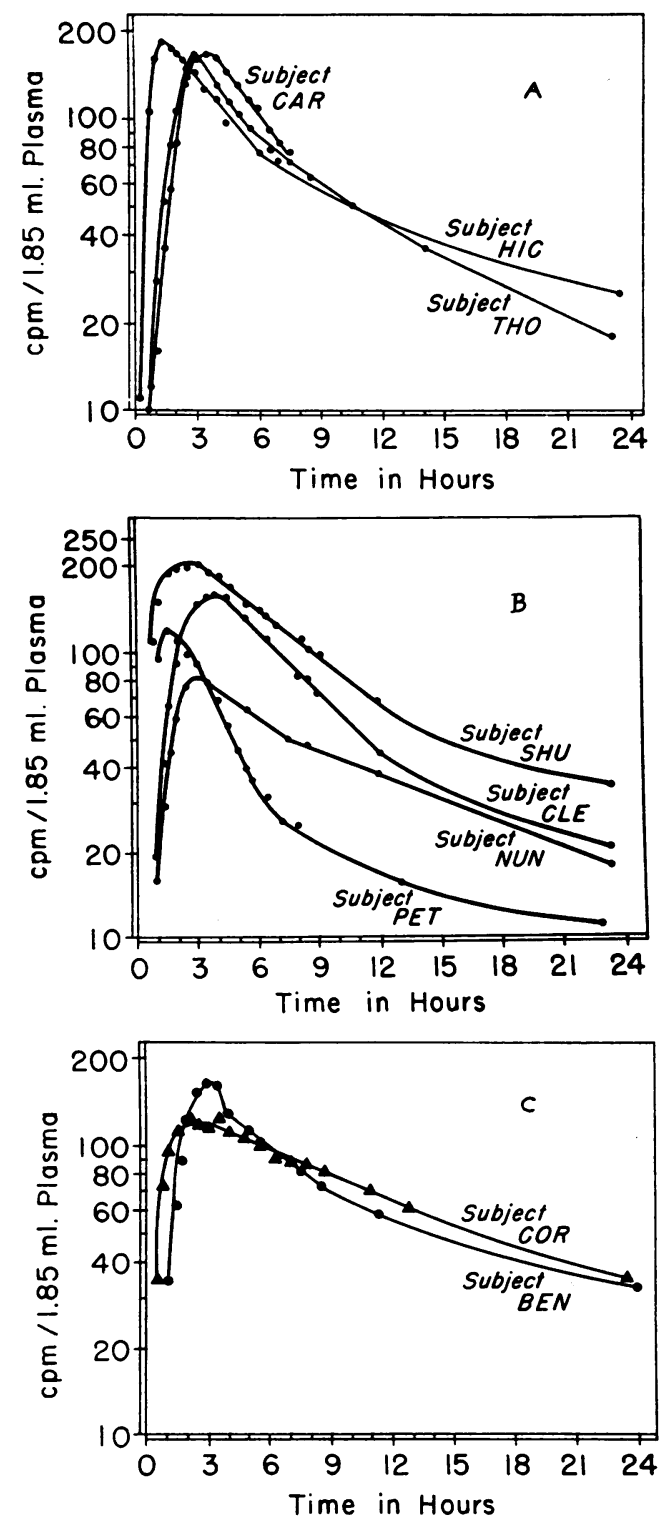

Fig. 1. Plasma triglyceride activity after intravenots injection of $\mathrm{C}^{14}$-Palmitate. Triglyceride activity represented by ascending limb and initial straight downslope only (see text).

radiopalmitate, $400 \mathrm{ml}$ of blood was withdrawn and analyzed for TG and FFA activity. The blood was collected in a standard evacuated blood donor set containing acid citrate dextrose solution as anticoagulant and stored at $4^{\circ} \mathrm{C}$. There is no evidence that this procedure does or does not denature or alter lipoproteins. A known quantity of plasma obtained from this blood was reinjected into another subject several days later.

This study was repeated using another subject who served both as donor and as recipient. Plasma neutral fat activity was determined in both donors and recipients as described in the preceding section.

\section{RESULTS}

\section{Non-reinjection experiments}

A. Triglyceride activity. ${ }^{1}$ The results in nine normal persons are presented in graphs in which the logarithm of activity is plotted against time (Figure 1). After rapid intravenous administration of albumin-bound palmitic acid-1-C ${ }^{14}$, there was a delay of about 10 to 15 minutes before activity began to appear. After the initial delay just described, activity rose rapidly and reached a peak in 2 to 4 hours, a time when FFA activity was very small. In the constant infusion experiments, a longer time was required to reach peak activity; otherwise these were not different from the single injection experiments (see Table I). (The purpose of the constant infusion studies was to simplify the kinetic problem by attempting to produce a situation in which the specific activity of TG influx into plasma was constant. This was later found to be both unnecessary and impractical.) Thereafter activity declined relatively rapidly at first and then more slowly over the 24 hour period of observation. Initially there was a straight line exponential decline after attainment of peak plasma activity. There was some uncertainty about this in two studies (Figure 1C). In all cases there was a departure from linearity beginning, at the earliest, 6 hours after injection of palmitic acid-1-C ${ }^{14}$. Figure 2 shows activity in TG 1 and 2 hours after injection of FFA. At 1

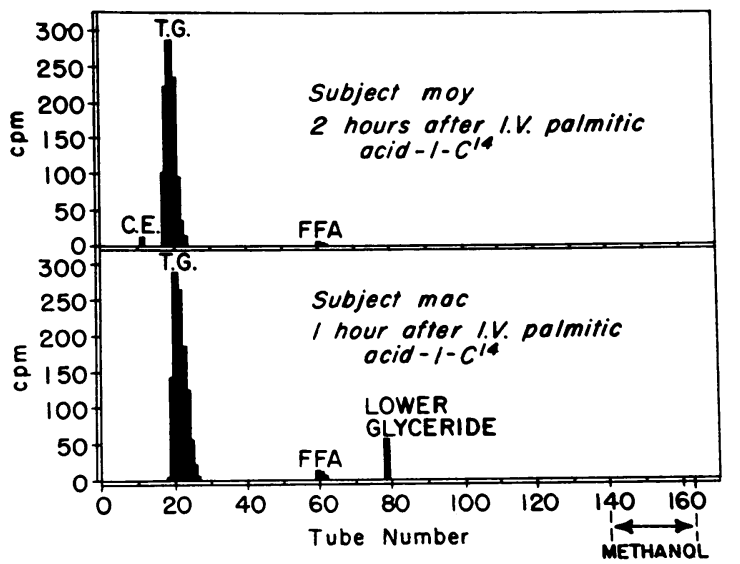

Fig. 2. Silicic acid chromatogram of plasma, 1 and 2 HOURS AFTER INJECTION OF $C^{14}$-PALMITATE BY GRADIENT ELUTION METHOD.

1 The curves described are a measure of total activity in plasma extracts. 
TABLE I

Observed and calculaied plasma triglyceride fatty acid contents and data used to obtain calculated levels in 9 subjects given palmitic acid-1-C14 *

\begin{tabular}{|c|c|c|c|c|c|c|c|c|c|c|c|c|c|}
\hline & 1 & 2 & 3 & 4 & 5 & 6 & 7 & 8 & 9 & 10 & 11 & 12 & 13 \\
\hline \multirow[t]{2}{*}{ Subject } & $\begin{array}{c}\text { Plasma } \\
\text { TGFA } \\
\text { content }\end{array}$ & $\begin{array}{c}\text { Calcu- } \\
\text { lated } \\
\text { plasma } \\
\text { TGFA } \\
\text { content }\end{array}$ & $\underset{\text { tion }}{\text { Devia- }}$ & $\begin{array}{c}x \\
\text { Plasma } \\
\text { TG } \\
\text { activity } \\
\text { at time } \\
t_{1}\end{array}$ & At $\underset{t_{1}}{y}$ & At $\underset{t_{1}}{z}$ & $\begin{array}{l}\text { Average } \\
\text { FFA } \\
\text { conc. }\end{array}$ & $\begin{array}{l}\text { FFA } \\
\text { flux }\end{array}$ & $\begin{array}{c}K \\
\text { Fractional } \\
\text { disappear- } \\
\text { ance rate } \\
\text { of plasma } \\
\text { TG per hr }\end{array}$ & $\begin{array}{c}F \\
\text { Fraction } \\
\text { of FA } \\
\text { label } \\
\text { appear- } \\
\text { ing in } \\
\text { plasma }\end{array}$ & $\begin{array}{c}a F \\
\text { Calcu- } \\
\text { lated } \\
\text { plasma } \\
\text { TG } \\
\text { influx }\end{array}$ & $\begin{array}{c}\text { FFA } \\
\text { adminis- } \\
\text { tered }\end{array}$ & $\begin{array}{l}\text { Calcu- } \\
\text { lated } \\
\text { plasma } \\
\text { volume }\end{array}$ \\
\hline & \multicolumn{2}{|c|}{$\begin{array}{c}\text { mmoles/totai } \\
\text { plasma vol }\end{array}$} & $\%$ & \multicolumn{3}{|c|}{$c p m / 1.85 \mathrm{ml}$ plasma } & mmoles $/ L$ & mmoles/hr & & & mmoles $/ \mathrm{hr}$ & $c p m$ & $m l$ \\
\hline CARt & 11.8 & 5.9 & -50 & 145 & 93.0 & 238.0 & 670 & 27.6 & 0.217 & 0.046 & 0.425 & $6.7 \times 10^{6}$ & 2,290 \\
\hline THO† & 6.5 & 5.9 & -9 & 94 & 118.5 & 212.5 & 700 & 29.0 & 0.232 & 0.047 & 0.458 & $5.86 \times 10^{6}$ & 2,300 \\
\hline BEN† & 12.0 & 9.8 & -19 & 70 & 110.5 & 180.5 & 450 & 26.1 & 0.123 & 0.046 & 0.400 & $6.12 \times 10^{6}$ & 2,900 \\
\hline NUN† & 30.1 & 20.5 & -35 & 45 & 63.5 & 108.5 & 600 & 50.3 & 0.115 & 0.059 & 0.783 & $5.51 \times 10^{6}$ & 4,670 \\
\hline CLE† & 15.3 & 24.0 & +50 & 130 & 91.9 & 221.9 & 700 & 34.3 & 0.165 & 0.093 & 1.067 & $4.84 \times 10^{6}$ & 3,270 \\
\hline HIC $\ddagger$ & 9.9 & 10.4 & +4.5 & 77 & 138.6 & 215.6 & 650 & 30.3 & 0.201 & 0.069 & 0.695 & $4.62 \times 10^{6}$ & 2,950 \\
\hline SHU‡ & 15.5 & 13.1 & -14 & 90 & 164.0 & 254.0 & 500 & 26.2 & 0.124 & 0.069 & 0.542 & $6.78 \times 10^{6}$ & 2,910 \\
\hline COR & 17.0 & 18.6 & +9 & 106 & 49.2 & 155.2 & 330 & 26.9 & 0.078 & 0.078 & 0.627 & $4.32 \times 10^{6}$ & 3,400 \\
\hline PET & 4.7 & 5.0 & +7 & 39 & 104.5 & 143.5 & 1,000 & 46.6 & 0.347 & 0.042 & 0.646 & $5.13 \times 10^{6}$ & 2,330 \\
\hline
\end{tabular}

* For explanation of $x, y$ and $z$, see text. Columns 1 and 2 give measured and calculated triglyceride concentrations; columns 4-13 give data used to obtain calculated levels in column 2 by methods described in the text.

$\dagger$ Constant infusion studies
‡ Single injection studies.

hour no activity was detected in cholesterol esters, and at 2 hours 1.5 per cent of the total activity was found in this fraction.

B. $\mathrm{CO}_{2}$ activity. Figure 3 illustrates the appearance of $\mathrm{CO}_{2}$ activity in expired air in relation to palmitic acid and TG activity during a constant infusion experiment and also illustrates the fact that peak expired $\mathrm{CO}_{2}$ labeling occurs before plasma TG peak activity.

C. Phospholipids. Table II shows the amount of activity in phospholipids at various intervals after the beginning of the studies. These determinations were performed on separate samples of plasma by silicic acid chromatography using

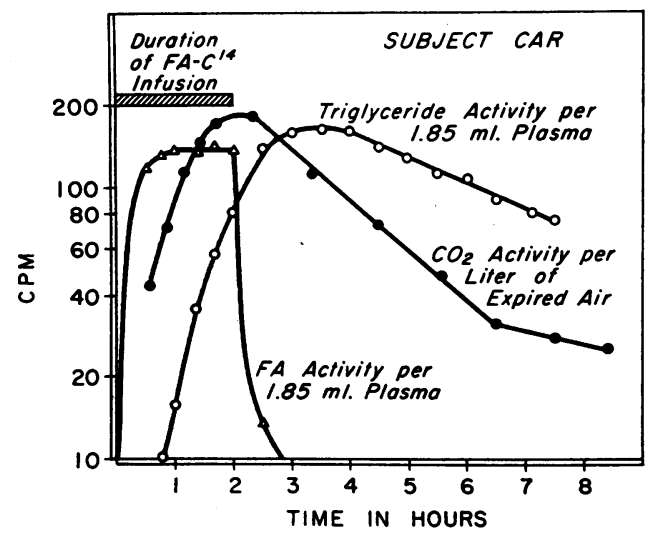

Fig. 3. Appearance of $\mathrm{CO}_{2}$ ACtivity In EXPIREd AIR IN RELATION TO PLASMA FREE FATTY ACID AND TRIGLYCERIDE ACTIVITY. methanol: chloroform $(1: 2, \mathrm{vol} / \mathrm{vol})$ for extraction of plasma.

D. Cholesterol esters. Figure 4, a representative example performed as a separate study, shows the appearance of label in cholesterol esters. Activity is initially small, but in 24 hours is responsible for a significant portion of the activity of the extracts. This can be ascertained by comparing the amount of cholesterol ester activity in Figure 4 with the residual total activity in Figure $1, \mathrm{~A}$ and $\mathrm{B}$.

E. Free cholesterol. Activity in free cholesterol constituted only a fraction of background activity.

TABLE II

Appearance of phospholipid activity in plasma after injection of palmitic acid-1-C

\begin{tabular}{ccc}
\hline Subject & $\begin{array}{c}\text { Time after } \\
\text { palmitic } \\
\text { acid-1-C.14 } \\
\text { administration }\end{array}$ & $\begin{array}{c}\text { Radio- } \\
\text { activity }\end{array}$ \\
\hline & hrs & $\begin{array}{c}\text { cpm per 1.85 } \\
\text { ml plasma }\end{array}$ \\
CAR & 1 & 20.4 \\
& 2.5 & 39 \\
& 5.5 & 41
\end{tabular}

THO

$\begin{array}{cc}1 & 19 \\ 3 & 20 \\ 5.5 & 26 \\ 22.5 & 40\end{array}$

CLE

$\begin{array}{lr}1 & 5 \\ 3 & 17 \\ 5.5 & 60\end{array}$




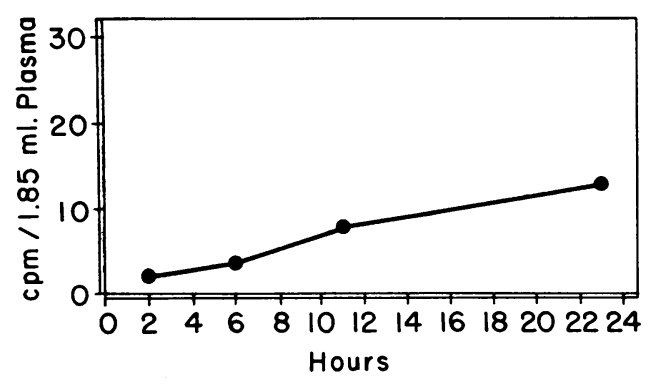

Fig. 4. Appearance of cholesterol ester activity AFTER INTRAVENoUS PALMitic ACID-1- $C^{14}$.

F. Oleic acid. The early part of the curves obtained (Figure 5 is a representative example) resembled the curves seen in the palmitic acid studies. The fractional turnover rates for the early TG portions of the curves were 0.225 and 0.314 per hour, respectively. Later the form of the curve was different. Duplicate determinations on separate samples of plasma revealed identical results. It is also quite clear from inspection that the amount of oleate label converted to TGFA label and the rate of disappearance are in the same range as these values for palmitate. Silicic acid chromatography of plasma obtained at 9 hours revealed that the difference in appearance between this curve and the palmitic acid curve is accounted for by a greater amount of activity in cholesterol esters. This is consistent with the known fact that cholesterol esters contain greater amounts of unsaturated acids (8).

$G$. Stearic acid. The form of the stearic acid curve resembled those for palmitic acid. The decline of radioactivity after attainment of peak ac-

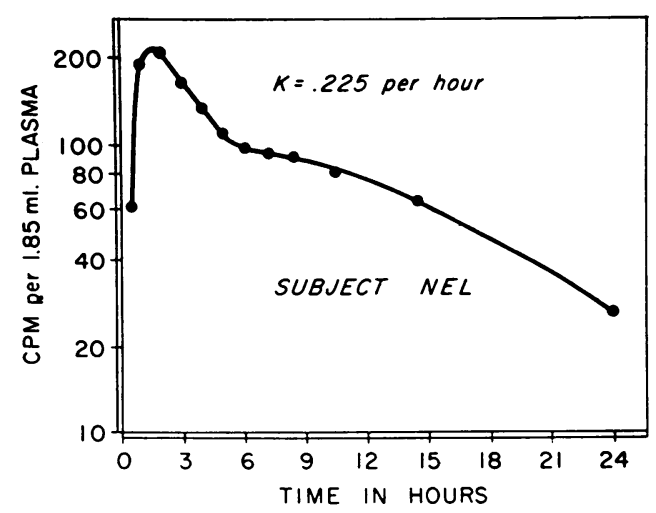

Fig. 5. Plasma triglyceride activity after injecTION OF $\mathrm{C}^{14}$-OLEATE. Triglyceride activity represented by ascending limb and initial straight downslope only (see text).

tivity was a straight line on the semilogarithmic plot and the fractional turnover rate was 0.146 per hour, a value which fell within the range of values obtained for palmitic acid. The curve was different in that maximal activity was only $51 \mathrm{cpm}$ per $1.85 \mathrm{ml}$ plasma, a value which was considerably lower than any obtained for palmitic acid (see Figure 1).

\section{Reinjection experiments}

The results of the two reinjection studies are summarized in Table III. In the first study, results indicated that the volume of distribution of the reinjected triglycerides was 25 per cent greater than the calculated plasma volume based on 40 $\mathrm{ml}$ plasma per $\mathrm{kg}$ body weight. The rate of disappearance of TG activity in the recipient was very

TABLE III

Plasma triglyceride fractional disappearance rates in reinjection sludies and related da!a

\begin{tabular}{|c|c|c|c|c|c|}
\hline & $\begin{array}{c}K \\
\text { Fractional } \\
\text { disappearance } \\
\text { rate of plasma } \\
\text { TG per hour }\end{array}$ & $\begin{array}{c}\text { No. of } \mathrm{cpm} \\
\text { injected }\end{array}$ & $\begin{array}{c}\text { Estimated } \\
\text { plasma } \\
\text { volume* }\end{array}$ & $\begin{array}{l}\text { Expected } \\
\text { activity at } \\
\text { ¿ero time }\end{array}$ & $\begin{array}{l}\text { Actual } \\
\text { extrapolated } \\
\text { activity at } \\
\text { zero time }\end{array}$ \\
\hline \multicolumn{6}{|l|}{ Expt. 1: } \\
\hline Donor & 0.422 & & & & \\
\hline Recipient & 0.402 & 26,300 & 3,000 & $\begin{array}{r}43.8 \mathrm{cpm} \text { per } \\
5 \mathrm{ml} \text { plasma }\end{array}$ & $\begin{array}{l}35 \mathrm{cpm} \text { per } \\
5 \mathrm{ml} \text { plasma }\end{array}$ \\
\hline \multicolumn{6}{|l|}{ Expt. 2: } \\
\hline \multicolumn{6}{|l|}{ Donor } \\
\hline Recipient & 0.282 & 17,050 & 2,200 & $\begin{array}{l}77.5 \mathrm{cpm} \text { per } \\
10 \mathrm{ml} \text { plasma }\end{array}$ & $\begin{array}{l}59 \mathrm{cpm} \text { per } \\
10 \mathrm{ml} \text { plasma }\end{array}$ \\
\hline
\end{tabular}

* Estimated plasma volume based on $40 \mathrm{ml}$ per $\mathrm{kg}$ body weight. 


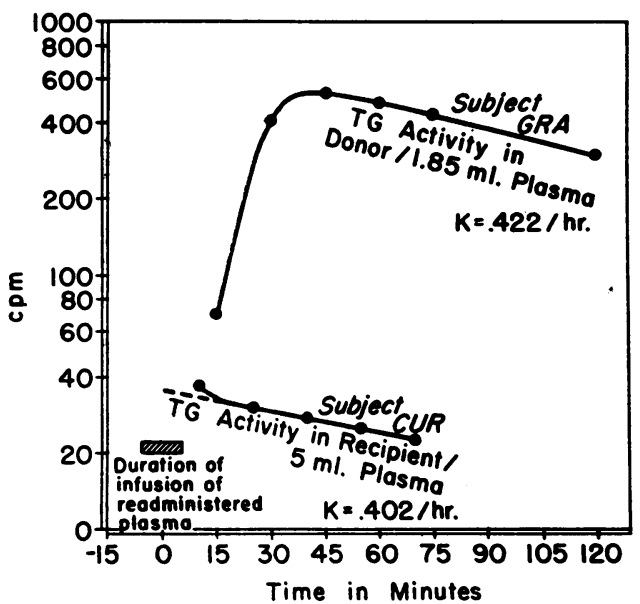

Fig. 6. Plasma triglyceride activity in Reinjection STUDY.

close to the initial rate of TG disappearance after attainment of peak activity in the donor (Figure 6 ), but in both donor and recipient was more rapid than in the non-reinjection studies. When the experiment was repeated using the same individual as donor and recipient, only the rate of decay of the reinjected material could be observed, and confirmed the previous findings that the volume of distribution corresponds rather closely (25 per cent greater) to the plasma volume and that the rate of disappearance was of the same order of magnitude observed in the non-reinjection experiments. Uptake of labeled TG during the period of infusion and possible equilibration with tissue TG are factors which may have been responsible for an overestimation of the size of the TG pool. In our first reinjection experiment the fractional disappearance rate was 0.402 per hour, a rate which was close to but higher than those found in our non-reinjection studies. However, in the second reinjection experiment, the fractional disappearance rate was 0.282 per hour, a value which fell within the range of the non-reinjection studies.

\section{INTERPRETATION}

\section{Background}

The studies in man of Fredrickson and Gordon (9) demonstrated that a portion of intravenously administered $\mathrm{C}^{\mathbf{1 4}}$-labeled fatty acids is later found in plasma as labeled neutral fat. There is good evidence that this conversion is effected in liver.
Thus Stein and Shapiro (10), and Laurell (11) found that this organ removed intravenously administered radiopalmitate and that activity was found in liver triglycerides and then in plasma triglycerides. In the experiments of Laurell, the specific activity of liver and plasma triglycerides appeared to be the same so that these findings were thought to be compatible with the concept that fasting plasma triglycerides might be entirely derived from liver triglycerides. On the other hand, Stein and Shapiro believed that liver triglycerides mix with a larger triglyceride pool from depots (10).

\section{Discussion of results}

A. Reinjection experiments. The observations in man made in this study differ from those in the rat in at least two obvious particulars. First, the rate of disappearance in the non-reinjection studies of Laurell is more rapid than that in man. Second, the rate of disappearance in the rat of reinjected fasting triglycerides is much more rapid than in the non-reinjection experiments (11). In our own studies this difference was either not observed or remained in doubt (see Figure 6 and compare $K$ values in Tables II and III). However, unpublished observations in this laboratory in dogs resemble those made in the rat by Laurell. From our own reinjection studies we conclude that the rate of disappearance resembled a simple first order decay and that the volume of distribution of the reinjected TG approximated the calculated plasma volume.

B. Non-reinjection experiments. The activity in an extract containing triglycerides, cholesterol esters, free cholesterol and some phospholipids is considered, on the basis of silic acid chromatographic studies, to represent triglyceride activity alone during the ascending portion of the curve and during the initial descending portion of the curve which forms a straight line on the semilogarithmic plot. Furthermore, the straight logarithmic downslope is considered to be representative of the disappearance of a single substance. This would be valid except in the unusual circumstance in which two or more different substances happened to be disappearing at the same fractional rate. The departure from linearity which occurs several hours after injection of labeled fatty acid 
can be explained either by recirculation of labeled triglyceride or by the appearance of another labeled material. The results, in fact, demonstrate the late appearance of activity augmenting the residual TG activity, both in cholesterol esters and in phospholipids.

\section{Current hypothesis}

The purpose of the studies on the relationship of $\mathrm{C}^{14}$-labeled fatty acids to plasma $\mathrm{C}^{14}$-labeled triglycerides was to evaluate the quantitative significance of this conversion in man.

As stated previously, it is believed, from the data of Laurell (11), Stein and Shapiro (10), and Hillyard, Cornelius and Chaikoff (12), that fatty acid is converted to triglyceride in the liver and that plasma TG may be in equilibrium with liver TG (11). However, in the present investigation, since only plasma levels were measured, it will be necessary to limit the interpretation arbitrarily to events occurring in the plasma pool even though this pool might be only a fraction of a larger TG pool with which TG equilibrates rapidly. The fractional rate of disappearance of TG-C ${ }^{14}$ calculated from the TG activity curves is interpreted to represent the rate of disappearance of fasting plasma triglycerides in general. It should be stated in this regard that TG molecules in blood are composed of a mixture of fatty acids (13) and are believed to be removed intact by adipose tissue in vitro (14). Another point in support of the hypothesis that TG is removed intact is the observation in this study that both oleic and palmitic acids, the two most abundant compounds in this class, behaved in the same way and fell into the same range of disappearance rates from plasma. It is also known that lower glycerides do not normally appear in plasma (3). Since TG molecules are composed of a mixture of fatty acids, one would anticipate that lower glycerides would be present in plasma if the rate of disappearance were more rapid for one species of fatty acid than for another. Futhermore, it is assumed that the calculated fractional rate is also the rate of disappearance for TG throughout the course of the study, especially during the time of increasing triglyceride activity.

Another important assumption, relating to the method of interpretation which follows, involves the concept that once peak plasma triglyceride ac- tivity has been reached and the straight exponential downward decay begins, no further net addition of TG label into the TG pool takes place. The word "net" is emphasized since a straight line could be observed if plasma TG equilibrates rapidly with other $T G$ pools, i.e., if plasma $T G$ were part of a larger TG pool from which TG disappearance proceeded at a uniform rate. The question that this investigation attempts to answer does not concern the total magnitude of conversion of FFA to TGFA, but whether enough FFA becomes TGFA to account for the observed TG concentration in plasma. The concept of "no further net addition" is justified by the following arguments. First, further net entry of label would alter linearity. Second, a model which fits the observed data was based on the observed rate of TG decay and a rate of entry into plasma also approximated from the data. The following equation was derived:

$$
x=\frac{k_{1} z_{0}}{k_{2}-k_{1}}\left(e^{-k_{1} t}-e^{-k_{2} t}\right)
$$

where $x$ is plasma TG activity, $k_{1}$ is the rate of disappearance from the pool emptying into plasma, $k_{2}$ is the plasma TG fractional disappearance rate, and $z_{0}$ is the amount of TG label released or equilibrating into plasma (15). Plotting this equation with values approximating those obtained experimentally gives a curve resembling the experimental curves. This equation, furthermore, is based on the assumption that release of TG label into plasma is exponential with time, an assumption which is consistent with the form of the increasing limb of the triglyceride activity curves. Third, inspection of the curves indicates that label equilibrates very rapidly at first and that this rapid rate of entry slows promptly and, therefore, the net influx of label would be expected to become rapidly insignificant and approach zero. The consequence of this would be that only the rate of disappearance of label would be manifest and that this picture would be uncomplicated by further addition of labeled material.

In addition to the foregoing, two other facts are pertinent to the discussion. First, plasma FFA is composed of about 50 per cent oleic acid, 30 per cent palmitic acid, 8 per cent stearic acid and several per cent of various other fatty acids 


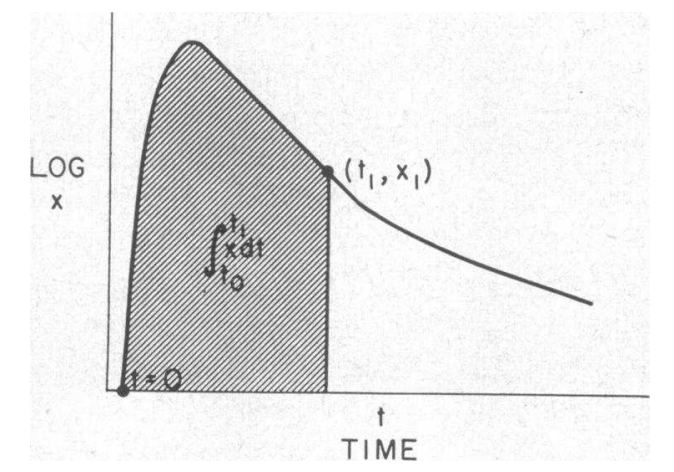

Fig. 7. Diagrammatic representation of data illustrating method of calculation. Explanation in text.

(16). Second, the fatty acid composition of triglycerides in plasma is very similar-approximately 40 per cent oleic, 30 per cent palmitic, 10 per cent linoleic, 5 per cent stearic, 5 per cent palmitoleic and varying smaller amounts of other fatty acids (8).

The presentation which follows is not intended to represent an accurate description of the kinetics of the individual studies but serves to indicate in a general way the magnitude of the conversion of FFA to plasma TG and to suggest that plasma FFA are the major source of plasma TG. The fractional disappearance rate and volume of distribution of plasma FFA, the plasma volume, and the values derived from these quantities were not experimentally determined but are representative of known normal values and may be the source of considerable error in individual studies. The interpretation is presented below.

In order to take into account the likely situation that plasma TG may be in part of a larger TG pool probably including liver TG, it is necessary to interpret the data in a manner which is independent of pool size. The following model is pictured. FFA label is converted to TGFA label which then equilibrates rapidly with all parts of the TG pool including plasma. This pool may be the same size or larger than the plasma pool. Disappearance then proceeds at a uniform rate from the entire pool.

In Figure 7, the logarithm of plasma TG activity, $x$, is plotted against time, $t$. Let $z=$ total TGFA activity (TGFA*) which has equilibrated with the plasma compartment, i.e., the total amount of administered fatty acid label $\left(\mathrm{FA}^{*}\right)$, which appeared in plasma as TGFA*. Let $y=$ amount of TGFA* which has left plasma from time $t=0$ to $t=t_{1} ; t_{1}$ is any point in time on the straight downlimb of the semilogarithmic plot. Let $M=$ amount of FA label administered. Let $F=$ fraction of $\mathrm{FA}$ label converted to plasma TGFA*. $F$ equals $z / M$. Let $a=$ endogenous FFA turnover rate or flux. Let $k=$ fractional disappearance rate of plasma TG. Then at any time $t$, on the straight downlimb of the semilogarithmic plot, $z=x-y ; d y$, the amount of TGFA* which has left plasma during an interval of time, $d t,=k x d t$, and the total amount of TFGA*, $y$, which has left the

plasma from $t=0$ to $t=t_{1}=\int_{t_{0}}^{t_{1}} k x d t$

and $z=x-k \int_{t_{0}}^{t_{1}} x d t$

But $F=z / M=\left[x-k \int_{t_{0}}^{t_{1}} x d t\right] / M$.

The amount of TGFA formed per hour which enters plasma equals the fraction of $\mathrm{FA}$ label converted to plasma TGFA* times the endogenous FFA turnover rate. This equals $F a$, which equals $a / M\left[x-k \int_{t_{0}}^{t_{1}} x d t\right]$.

If TGFA enters plasma at the rate of $F a$ millimoles per hour and leaves at the fractional disappearance rate $k$, the anticipated plasma TGFA content which will be reached can be expressed by $\mathrm{Fa} / k\left(1-e^{-k t}\right)(17)$.

When $t=\infty$, plasma TFGA content will equal $F a / k$; or, substituting $F a=a / M[x-k$ $\left.\times \int_{t_{0}}^{t_{1}} x d t\right]$, plasma TGFA content $=a / M k$ $\times\left[x-k \int_{t_{0}}^{t_{1}} x d t\right]=a / M\left[x / k-\int_{t_{0}}^{t_{1}} x d t\right]$. The integral $\int_{t_{0}}^{t_{1}} x d t$ is the area under the shaded portion of the curve. This area was determined in these studies by the trapezoid rule. The area depicted in the diagram is, of course, not correct since it is drawn under semilogarithmic plot. The correct area would be represented by the equivalent area drawn under an arithmetic plot. In practice the semilogarithmic diagram was used to facilitate the 
measurement of $k$ and to insure that $x_{1}$ is measured on the straight semilogarithmic downslope.

Note that when $t_{1}=\infty, x=0$ and plasma TGFA $=a / M \int_{t_{0}}^{t_{1}} x d t$. The expression $1 / M$ $\times \int_{t_{0}}^{t_{1}} x d t$ equals the area under the TGFA curve from $t=0$ to $t=\infty$ divided by the total amount of FA label administered. ${ }^{2}$

In calculating the data with the equations presented, the following factors were taken into account: 1) Plasma volumes were calculated on the basis of $40 \mathrm{ml}$ plasma per $\mathrm{kg}$ body weight (18). 2) It was assumed that the fraction of palmitic acid-1-C ${ }^{14}$ converted to TGFA* is representative

${ }^{2}$ Since the writing of this paper an alternative method of analyzing the data has been proposed. The derivation which follows is more general in nature, and under certain circumstances requires no knowledge concerning the rate of disappearance of TGFA.

Let $\omega=$ time of introduction of labeled FA. Let $M=$ total dose of labeled FA. Let $f(t-\omega)=$ fraction of a given quantity of FFA which enters plasma at time $\omega$ and which is to be found with plasma TGFA pool at time $t$ (a steady state is assumed). Let $m(\omega)=$ plasma FFA flux (rate of entry of FFA into plasma). Then, the quantity of FFA which entered plasma during time interval $d \omega$ is $m(\omega) d \omega$.

At time $t$ from the time this quantity, $m(\omega) d \omega$, entered, the quantity $f(t-\omega) m(\omega) d \omega$ would be found in the plasma TGFA pool, or adding up for all previous time intervals we obtain:

$$
\text { total plasma TGFA }=\int_{-\infty}^{t} f(t-\omega) m(\omega) d \omega
$$

The substitution $\Omega=t-\omega$ transforms this into the equivalent expression :

$$
\text { total plasma TGFA }=\int_{0}^{\infty} m(t-\Omega) f(\Omega) d \Omega
$$

If the rate of entry of FFA into the plasma is constant, say $m(\omega)=a$, then the quantity of TGFA present at time $t$ will be :

$$
\text { total TGFA }=a \int_{0}^{\infty} f(\Omega) d \Omega
$$

The integral $\int_{0}^{\infty} f(\Omega) d \Omega$ is the total area under the plasma

TGFA curve divided by the total initial dose of labeled fatty acid. It can be seen immediately that the equation

TGFA $=a \int_{0}^{\infty} f(\Omega) d \Omega$ is identical with the equation presented in the main text when $t=\infty$.

The alternative equation for practical application requires extrapolation of the straight semilogarithmic downlimb of the TGFA* curves to infinity in order to measure the required area. of fatty acids as a whole. Whereas this is not strictly true it is a very reasonable approximation, since the fatty acid composition of plasma FFA and plasma TGFA is almost identical $(8,16)$ and since oleic and palmitic acids behaved similarly. 3 ) Endogenous hourly FFA turnover rate, $a$, was estimated from the average plasma FFA level during the period of TGFA* formation by using a fractional disappearance rate of 0.3 per minute (9). The plasma volume was taken as the volume of distribution of FFA, although the latter volume may be somewhat greater than the former $(4,9) .4)$ Extraction efficiency of 87 per cent for FFA and 95 per cent for TG was taken into account.

The data and method of calculations outlined above were used to estimate an anticipated plasma triglyceride content for each subject based on a rate of TG formation calculated from the isotope data. The calculated levels would represent a minimal estimate if recirculation of TGFA occurs. These anticipated or theoretically expected levels derived from the tracer data were compared with the actual TGFA plasma contents in each sul-ject. The actual TG contents with which the calculated levels were compared were the means of several levels obtained during the period of formation of labeled TGFA. During this time there was little fluctuation in TG concentration. The data and calculations appear in Table I. Plasma TG content was calculated from the determinations by using a mol wt of 900 . It can be seen (columns 1, 2 and 3) that the calculated approximations range from 50 per cent less to 50 per cent more than the measured levels and that six fall within 19 per cent of the measured levels. The same results can be obtained in a somewhat different manner: the plasma TGFA efflux is the product of $k$ and TGFA content and this efflux can be compared with $a F$, the calculated rate of entry of plasma TGFA. Under these circumstances the results are unaltered. Since calculated TGFA flux into plasma approximates endogenous TGFA flux out of plasma, it would appear from these calculations that all three fatty acids of triglyceride turn over and that the majority of plasma TG originated from FFA. That the major fatty acids of TG turn over at the same rate is strongly suggested not only from a comparison of the results with palmitic acid-1- $\mathrm{C}^{14}$ 
and oleic acid-1-C ${ }^{14}$, but also from data of Rodbell showing that TG is removed intact by adipose tissue in vitro (14).

A point which should be emphasized is that the results, suggesting that plasma FFA conversion to plasma TGFA is sufficient to account for plasma TG concentration, would be the same if these calculations were based on a unit volume of plasma ; i.e., the interpretation is independent of pool size. Furthermore, the value obtained for the fraction of radiopalmitate converted to plasma TGFA applies only to the initial equilibration of label into plasma. The actual fraction of FFA converted to TGFA might be considerably in excess of this amount if one considers, as Laurell claims (11), that plasma TG specific activity might well be the same as that in liver and that liver TG probably greatly exceeds plasma TG.

\section{DISCUSSION AND CONCLUSIONS}

An important concomitant of the present interpretation is the concept that one of the factors relating directly to the plasma TG level is the magnitude of plasma FFA turnover rate. This idea finds support in observations relating plasma FFA concentration to plasma TG concentration. For example, Havel (19) found that administration of glucose, which causes a fall in plasma FFA, also causes a fall in plasma TG concentration. Dury and Treadwell (20) showed that epinephrine which raises plasma FFA concentration also raises plasma TG concentration. Both diabetes and starvation produce a rise in plasma FFA and plasma TG.

Another point which should be made is that the present study does not distinguish actual triglyceride formation from a process which exchanges one TGFA for another from FFA. There is, however, suggestive evidence from the studies of others that FFA may not be released from the liver. The large positive FFA arteriovenous difference of the blood perfusing liver (21) suggests that such an exchange may not be the only mechanism concerned. Stein and Shapiro were unable to find significant amounts of FFA label after uptake of $\mathrm{C}^{14}$-triglycerides by rat liver (22). This last point is, however, difficult to evaluate because liver FFA is rapidly diluted by a large FFA flux from plasma. It has also been shown that plasma triglycerides are removed by adipose tissue in vitro $(14,23)$, and that only a fraction of administered $\beta$-lipoprotein triglyceride is taken up by liver (22). If plasma triglyceride undergoes a fate other than recirculation to liver and if, as has been shown in these isotopic studies, the majority, and perhaps all plasma TG originates from liver, it would be unlikely that incorporation of FFA into TGFA would be entirely an exchange phenomenon. However, proof of this contention is not possible at present.

It is also necessary to point out evidence concerning the importance of the liver in the incorporation of FFA into TGFA and the lack of participation of extrahepatic tissues in contributing to the fasting triglyceride via an FFA precursor. Such evidence is forthcoming not only from animal studies already mentioned but also from the recent observation by Havel and Goldfien (24) that hepatectomy in dogs abolished the conversion of FFA to TGFA.

It is concluded from the data presented and from the interpretation of these data that plasma FFA is a major source of fasting plasma triglyceride. The calculations, furthermore, strongly suggest that FFA is involved in a process in which all three fatty acids of triglyceride turn over and that FFA is therefore the preponderant source of fasting TGFA.

\section{SUMMARY}

Observations have been made on the incorporation of injected $\mathrm{C}^{14}$-labeled fatty acids into triglycerides, cholesterol esters, free cholesterol and phospholipids.

An estimate was made of the quantitative aspect of the formation of triglyceride label. The results of this estimate are consistent with the hypothesis that a major fraction of fasting plasma triglyceride is derived from plasma fatty acids.

\section{ACKNOWLEDGMENTS}

The technical assistance of Mrs. Anna B. Ferguson, Miss Helen L. Hilderman, Mrs. Gray D. Long, Mrs. Sue H. Peters, Miss Corinna Thomas, Mrs. Gitta W. Jackson and $\mathrm{Mr}$. Albert Eaton is gratefully acknowledged. In addition, we would like to thank Dr. Thomas M. Gallie, Jr., Department of Mathematics, Duke University, for his invaluable assistance in reviewing the mathematical problems discussed. 


\section{REFERENCES}

1. Dole, V. P. A relation between non-esterified fatty acids in plasma and the metabolism of glucose. J. clin. Invest. 1956, 35, 150 .

2. Borgström, B. Investigation of lipid separation methods. Separation of phospholipids from neutral fat and fatty acids. Acta physiol. scand. 1952, 25, 101.

3. Hirsch, J., and Ahrens, E. H., Jr. The separation of complex lipide mixtures by the use of silicic acid chromatography. J. biol. Chem. 1958, 233, 311.

4. Friedberg, S. J., Klein, R. F., Trout, D. L., Bogdonoff, M. D., and Estes, E. H., Jr. The characteristics of the peripheral transport of $\mathrm{C}^{14}$-labeled palmitic acid. J. clin. Invest. 1960, 39, 1511.

5. Van Handel, E., and Zilversmit, D. B. Micromethod for the determination of serum triglycerides. $\mathrm{J}$. Lab. clin. Med. 1957, 50, 152.

6. Rapport, M. M., and Alonzo, N. Photometric determination of fatty acid ester groups in phospholipides. J. biol. Chem. 1955, 217, 193.

7. Fredrickson, D. S., and Ono, K. An improved technique for assay of $\mathrm{C}^{14} \mathrm{O}_{2}$ in expired air using the liquid scintillator counter. J. Lab. clin. Med. 1958, $51,147$.

8. Hallgren, B., Stenhagen, S., Svanborg, A., and Svennerholm, L. Gas chromatographic analysis of the fatty acid composition of the plasma lipids in normal and diabetic subjects. J. clin. Invest. 1960, 39, 1424.

9. Fredrickson, D. S., and Gordon, R. S., Jr. The metabolism of albumin bound $\mathrm{C}^{\mathbf{1 4}}$-labeled unesterified fatty acids in normal human subjects. $\mathrm{J}$. clin. Invest. 1958, 37, 1504.

10. Stein, Y., and Shapiro, B. Assimilation and dissimilation of fatty acids by the rat liver. Amer. J. Physiol. 1959, 196, 1238.

11. Laurell, S. Recycling of intravenously injected palmitic acid-1- $\mathrm{C}^{14}$ as esterified fatty acid in the plasma of rats and turnover rate of plasma triglycerides. Acta physiol. scand. 1959, 47, 218.
12. Hillyard, L. A., Cornelius, C. E., and Chaikoff, I. L. Removal by the isolated rat liver of palmitate-1-C $\mathrm{C}^{\mathbf{1 4}}$ bound to albumin and of palmitate-1- $\mathrm{C}^{14}$ and cholesterol-4-C $\mathrm{C}^{14}$ in chylomicrons from perfusion fluid. J. biol. Chem. 1959, 234, 2240.

13. Reiser, R., Bryson, M. J., Carr, M. J., and Kuiken, K. A. The intestinal absorption of triglycerides. J. biol. Chem. 1952, 194, 131.

14. Rodbell, M. The removal and metabolism of chylomicrons by adipose tissue in vitro. J. biol. Chem. 1960, 235, 1613.

15. Zilversmit, D. B. The design and analysis of isotope experiments. Amer. J. Med. 1960, 29, 832.

16. Dole, V. P. Fractionation of plasma non-esterified fatty acids. Proc. Soc. exp. Biol. (N. Y.) 1956, 93, 532 .

17. Jokipii, S. G., and Turpeinen, O. Kinetics of elimination of glucose from the blood during and after a continuous intravenous injection. $\mathrm{J}$. clin. Invest. 1954, 33, 452.

18. Storaasli, J. P., Krieger, H., Friedell, H. L., and Holden, W. L. Use of radioactive iodinated plasma protein in study of blood volume. Surg. Gynec. Obstet. 1950, 91, 458.

19. Havel, R. J. Early effects of fasting and of carbohydrate ingestion on lipids and lipoproteins of serum in man. J. clin. Invest. 1957, 36, 855.

20. Dury, A., and Treadwell, C. R. Effect of epinephrine on plasma lipid components and interrelationships in normal and epileptic humans. J. clin. Endocr. $1955,15,818$.

21. Gordon, R. S., Jr., and Cherkes, A. Unesterified fatty acid in human blood plasma. J. clin. Invest. 1956, 35, 206.

22. Stein, Y., and Shapiro, B. Uptake and metabolism of triglycerides by the rat liver. J. Lip. Res. 1960 , $1,326$.

23. Stein, I., and Shapiro, B. The transport of lipids into adipose tissue. Metabolism 1954, 3, 539.

24. Havel, R., and Goldfien, A. Role of liver and extrahepatic tissues in plasma fatty acid metabolism. Clin. Res. 1960, 8, 141. 